\title{
1-Chloro-1,3-butadiene Copolymers IV. Bulk Copolymerization of 1-Chloro-1,3-butadiene with Styrene
}

\author{
Shinzo KoHJIYA, Masanori KuroKawa, Kazuo IWATA, \\ Yoshihiko IMOTO, Takashi ENOKI \\ and Shinzo YAMASHITA \\ Department of Chemistry, Kyoto Institute of Technology, \\ Matsugasaki, Kyoto 606, Japan
}

(Received June 23, 1983)

\begin{abstract}
In the bulk copolymerization of 1-chloro-1,3-butadiene (CB) and styrene (St) at $70^{\circ} \mathrm{C}$, the copolymerization rate, monomer reactivity ratio (MRR), and elementary reactions governing the molecular weight of the copolymer were studied. The rate of St polymerization decreased drastically by the addition of a small amount of $\mathrm{CB}$, suggesting a predominant crossover termination, $\left(k_{t_{12}}\right)$. MRRs found: $r_{\mathrm{CB}}=1.680$ and $r_{\mathrm{St}}=0.274$, hence $Q=1.80$ and $e=0.081$ for $\mathrm{CB}$. The molecular weight of copolymer and its polydispersity decreased with an increase in $\mathrm{CB}$ concentration, which is interpreted by a large $k_{t_{12}}$ value. When the CB concentration becomes large, termination between CB polymer radicals may also become important.

KEY WORDS Copolymerization / Monomer Reactivity Ratios /

Termination / Molecular Weight / Molecular Weight Distribution /

1-Chloro-1,3-butadiene / Styrene /
\end{abstract}

The copolymer of 1-chloro-1,3-butadiene (CB) and 1,3-butadiene (Bd) contains active chlorines which enable it to undergo various chemical modifications. ${ }^{1-3}$ These chlorines were estimated to be in 1,4-configurational $\mathrm{CB}$ units, ${ }^{3}$ as was also the case in $\mathrm{CB}$-styrene $(\mathrm{St})$ copolymer. ${ }^{4,5}$ Thus, CB copolymers are regarded as functional polymers, and their reactivities are considered interpretable from their chemical structure determined by monomer reactivity ratios (MRR), conversions, and the microstructure of CB units. Therefore, a complete characterization of the copolymer seems very important for using the CB copolymer as a functional polymer.

In a series of previous works, we attempted to characterize all aspects of poly(CB-co-St), i.e., not only the overall composition but compositional distribution, monomer sequence distributions and some other molecular properties as well. In the present work, co- polymerization of $\mathrm{CB}$ with $\mathrm{St}$ in the presence of 2,2'-azobisisobutyronitrile (AIBN) was studied to elucidate the reactivity of $\mathrm{CB}$ in connection with the molecular weight (MW) and molecular weight distribution (MWD) of poly(CB-co-St). Polymerizations were carried out in bulk to obtain pure samples. Additionally, since only a low MW copolymer was obtained by solution polymerization, ${ }^{5}$ bulk polymerization was preferred to solution polymerization. Monomer reactivity ratios were determined and the factors influencing the MW and MWD of the copolymer are discussed.

\section{EXPERIMENTAL}

\section{Materials}

CB was synthesized according to the method of Heasley and Lais ${ }^{6}$ : bp $65.5-67.5^{\circ} \mathrm{C}$ (lit $^{6}$ $66-68^{\circ} \mathrm{C}$ ); purity by gas-liquid chromatog- 
raphy, 98.6\%; IR and ${ }^{1} \mathrm{H}$ NMR spectra were fully consistent with CB. St, AIBN, and other reagents were purified by the usual methods.

\section{Polymerization}

Monomers and AIBN were introduced into a glass ampoule, which was connected to a vacuum system. The ampoule was subjected to degassing and sealed under high vacuum $\left(2 \times 10^{-5}\right.$ Torr $)$. The ampoule was placed in a bath maintained at $70^{\circ} \mathrm{C}$ and rotated at a speed of $24 \mathrm{~min}^{-1}$. Poly(CB-co-St) has been reported to be relatively unstable,,$^{5,7}$ probably due to $\mathrm{HCl}$ elimination from the backbone chain. Therefore, we used methanol containing a small amount of di- $n$-butyltin dilaurate to coagulate the copolymer. Even so, only freshly prepared copolymer was investigated.

\section{Polymer Characterization}

The number-average molecular weights of the polymers were determined by a Knauer membrane osmometer. The gel permeation chromatogram (GPC) was obtained on a Toyo Soda HLC-802UR equipped with refractive index (RI) and UV detectors: eluent, tetrahydrofuran; flow rate, $1 \mathrm{ml} \mathrm{min}^{-1}$; column temperature, $40^{\circ} \mathrm{C}$. Copolymer compositions were calculated from chlorine content determined by elemental analyses, and were in good agreement with those determined by ${ }^{1} \mathrm{H}$ NMR and thin-layer chromatography. ${ }^{5,8}$ Monomer reactivity ratios were evaluated by the Fineman-Ross method.

\section{RESULTS AND DISCUSSION}

\section{Copolymerization of $C B$ and $S t$}

Table I shows the results of the copolymerizations of $\mathrm{CB}$ and St made to stop at a conversion lower than $10 \%$ except No. 5 . Figure 1 gives the variation in percentage conversion per minute with monomer feed composition. The percentage conversion per

Table I. Bulk copolymerization of $\mathrm{CB}$ and St at $70^{\circ} \mathrm{C}^{\mathrm{a}}$

\begin{tabular}{|c|c|c|c|c|}
\hline \multirow{2}{*}{ Sample } & \multirow{2}{*}{$\frac{\begin{array}{l}\text { CB content in } \\
\text { monomer feed }\end{array}}{\mathrm{mol} \%}$} & \multirow{2}{*}{$\frac{\text { Time }}{\min }$} & \multirow{2}{*}{$\frac{\text { Conversion }}{\%}$} & \multirow{2}{*}{$\begin{array}{c}\begin{array}{c}\text { CB content } \\
\text { in copolymer }\end{array} \\
\mathrm{mol} \%\end{array}$} \\
\hline & & & & \\
\hline 1 & 0 & 20 & 7.40 & 0 \\
\hline 2 & 0.180 & 30 & 6.92 & 1.55 \\
\hline 3 & 0.450 & 30 & 6.13 & 2.11 \\
\hline 4 & 1.33 & 42 & 6.56 & 4.58 \\
\hline 5 & 2.90 & 180 & 11.7 & 9.75 \\
\hline 6 & 4.20 & 61 & 5.53 & 12.8 \\
\hline 7 & 7.10 & 240 & 9.33 & 19.7 \\
\hline 8 & 7.65 & 200 & 7.34 & 21.1 \\
\hline 9 & 9.44 & 121 & 7.47 & 24.4 \\
\hline 10 & 10.2 & 150 & 8.81 & 25.5 \\
\hline 11 & 17.3 & 150 & 5.64 & 38.5 \\
\hline 12 & 24.3 & 122 & 4.93 & 46.5 \\
\hline 13 & 33.0 & 178 & 7.14 & 55.7 \\
\hline 14 & 48.0 & 177 & 5.89 & 67.5 \\
\hline 15 & 64.0 & 211 & 6.43 & 76.9 \\
\hline 16 & 77.0 & 240 & 7.11 & 85.9 \\
\hline 17 & 89.0 & 240 & 6.43 & 90.0 \\
\hline 18 & 100 & 300 & 8.25 & 100 \\
\hline
\end{tabular}

${ }^{\text {a }}[$ Monomer $] /[\mathrm{AIBN}]=200$.

b Calculated from chlorine content. 


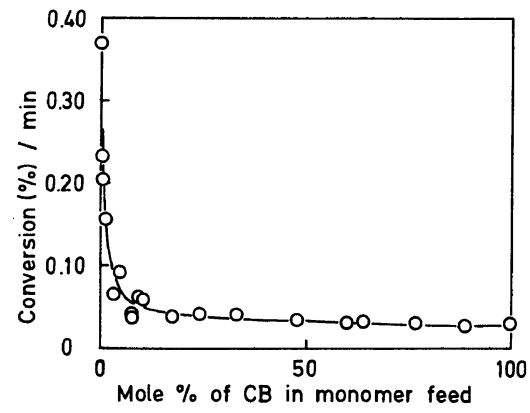

Figure 1. Rate of copolymerization of CB-St in bulk at $70^{\circ} \mathrm{C}$ [monomer] $/[\mathrm{AIBN}]=200$; rate is expressed by conversion $(\%) / \min ($ see Table I).

minute was, exactly speaking, not a rate but a substitute for rate when [AIBN] was maintained constant and the conversions kept low. The observed drastic variation in the copolymerization rate was similar to that of St-methyl

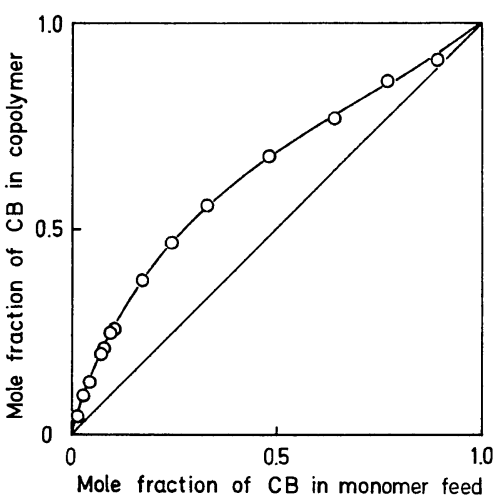

Figure 2. Copolymer composition curve of CB-St; see Table I.

methacrylate copolymerization at $60^{\circ} \mathrm{C}$ by AIBN, ${ }^{9}$ St-butyl acrylate, ${ }^{10}$ or St-1-pyridylbutadiene copolymerization. ${ }^{11}$ The copolymerization rate is expressed in the form ${ }^{9,12,13}$

$$
-\frac{\mathrm{d}\left(\left[\mathrm{M}_{1}\right]+\left[\mathrm{M}_{2}\right]\right)}{\mathrm{d} t}=\frac{R_{i}^{1 / 2}\left(r_{1}\left[\mathrm{M}_{1}\right]^{2}+2\left[\mathrm{M}_{1}\right]\left[\mathrm{M}_{2}\right]+r_{2}\left[\mathrm{M}_{2}\right]^{2}\right)}{\left(\delta_{1}^{2} r_{1}^{2}\left[\mathrm{M}_{1}\right]^{2}+2 \phi r_{1} r_{2} \delta_{1} \delta_{2}\left[\mathrm{M}_{1}\right]\left[\mathrm{M}_{2}\right]+\delta_{2}^{2} r_{2}^{2}\left[\mathrm{M}_{2}\right]^{2}\right)^{1 / 2}}
$$

where $r_{1}=k_{11} / k_{12}, r_{2}=k_{22} / k_{21}, R_{i}$ is the initiation rate, $\delta_{1}^{2}=k_{t_{1}} / k_{11}^{2}, \delta_{2}^{2}=k_{t_{2}} / k_{22}^{2}, \phi^{2}=$ $k_{t_{12}}^{2} / k_{t_{1}} k_{t_{2}}, k_{i j}(i, j=1$ or 2$)$ is the propagation rate constant for the reaction in which $j$ monomer adds to an $i$-radical, $k_{t_{i}}(i=1$ or 2$)$ is the rate constant for the mutual termination of two $i$-radicals, and $k_{t_{12}}$ is the crossover termination rate constant. The drastic decrease in rate of the above mentioned copolymerization systems was explained by assuming a much larger $k_{t_{12}}$ value than the $k_{t}$ 's of homopolymerizations, i.e., $\phi$ to be much larger than unity. $^{9-11}$

Figure 2 depicts the copolymer composition curve. By the Fineman-Ross method, the experimental points gave the following monomer reactivity ratios:

$$
r_{1}=0.274 \pm 0.069, \quad r_{2}=1.680 \pm 0.001
$$

where $M_{1}$ and $M_{2}$ refer to St and $C B$, respectively. The solid line in Figure 2 is that calculated from these values. The values are somewhat different from the previous results: $r_{1}=0.10, r_{2}=1.21$ for the solution polymerization at $70^{\circ} \mathrm{C}$ by $\mathrm{AIBN}^{5} ; r_{1}=0.23, r_{2}=1.02$ for the radiation-induced bulk polymerization. ${ }^{7}$ To poly(CB-co-St) produced in bulk by AIBN at $70^{\circ} \mathrm{C}$, our values are most pertinent. The $Q$ and $e$ values evaluated from $r_{1}=0.274$ and $r_{2}=1.680$ are:

$$
Q_{2}=1.80, \quad e_{2}=+0.081
$$

These are very reasonable values since CB is a conjugated monomer and chlorine is an electron-withdrawing substituent (for styrene, $\left.Q_{1}=1.00, e_{1}=-0.80\right) .{ }^{14}$

From the $r_{1}$ and $r_{2}$ values, $k_{12}>k_{11}$ and $k_{22}>k_{21}$. If $k_{11} \gg k_{22}$ is the case, the order, $k_{12}>k_{11} \gg k_{22}>k_{21}$ holds and the behavior shown in Figure 1 may be explained by these differences between constants. In other words, $\mathrm{CB}$ is a very reactive monomer, and hence $\mathrm{CB}$ polymer radical is very stable. This high stability of the $\mathrm{CB}$ polymer radical may cause the 
drastic decrease in the polymerization rate shown in Figure 1. However, the $k_{\mathrm{p}}$ value of $\mathrm{CB}\left(k_{22}\right)$ is not available, and it is not certain if $k_{11} \gg k_{22}$ is actually the case. A similar decrease in $\mathrm{MW}$ of poly(CB-co-St) can be better explained by the predominance of crossover termination. Therefore, the large $k_{t_{12}}$ seems a better assumption as has been estimated so far. $^{9-11}$

Decrease in Molecular Weight of Poly(CB-co$S t$ )

The MW of the poly(CB-co-St)s were measured on a membrane osmometer and the results are listed in Table II. The higher the CB content in monomer feed, the higher was the $\mathrm{CB}$ content in the copolymer and the smaller the MW. We can calculate the number-average degree of polymerization from MW data, taking the copolymer composition into account. The values are shown in the last column, and decrease with CB content. The GPC measurement on poly(CB-co-St) indicates that the MWD is an ordinary value in radical polymerization. Since the $\mathrm{MW}$ of poly $(\mathrm{CB}-\mathrm{co}-\mathrm{St})$ changes with $\mathrm{CB}$ concentration and concentration changes with conversion (see Figure 2), the MW of poly(CB-co-St) necessarily changes with conversion.

To ascertain the effects of $\mathrm{CB}$ concentration further, MW measurements on poly(CB-co$\mathrm{St}) \mathrm{s}$ produced at higher conversions were carried out. Table III shows the results. At these conversions, almost all $\mathrm{CB}$ was polymerized,

Table II. Molecular weights of poly(CB-co-St)s

\begin{tabular}{|c|c|c|c|c|}
\hline Sample $^{\mathrm{a}}$ & $\begin{array}{l}\begin{array}{l}\mathrm{CB} \text { content in } \\
\text { monomer feed }\end{array} \\
\mathrm{mol} \%\end{array}$ & $\frac{\begin{array}{c}\text { CB content } \\
\text { in copolymer }\end{array}}{\mathrm{mol} \%}$ & $\begin{array}{l}\text { Molecular } \\
\text { weight }^{\mathrm{b}}\end{array}$ & $\begin{array}{c}\text { Degree of } \\
\text { polymerization }\end{array}$ \\
\hline 1 & 0 & 0 & 72,300 & 695 \\
\hline 2 & 0.180 & 1.55 & 60,000 & 579 \\
\hline 3 & 0.450 & 2.11 & 46,700 & 451 \\
\hline 4 & 1.33 & 4.58 & 36,100 & 350 \\
\hline 6 & 4.20 & 12.76 & 25,700 & 253 \\
\hline 8 & 7.65 & 21.43 & 27,700 & 277 \\
\hline 9 & 9.44 & 24.43 & 23,300 & 235 \\
\hline 11 & 17.3 & 38.49 & 24,400 & 252 \\
\hline
\end{tabular}

a All samples are obtained at conversion lower than $10 \%$ (see Table I).

b Measured on a Knauer membrane osmometer in benzene at $37^{\circ} \mathrm{C}$. The results are estimated to be with an error of about $\pm 5.0 \%$.

c Calculated from molecular weight and copolymer composition.

Table III. Molecular weights of poly(CB-co-St)s obtained at higher conversions

\begin{tabular}{|c|c|c|c|c|}
\hline Sample & $\begin{array}{l}\begin{array}{l}\mathrm{CB} \text { content in } \\
\text { monomer feed }\end{array} \\
\mathrm{mol}^{\mathrm{o}} \%\end{array}$ & $\frac{\text { Conversion }}{\%}$ & $\begin{array}{c}\begin{array}{c}\text { CB content } \\
\text { in copolymer }\end{array} \\
\mathrm{mol}_{\%} / \mathrm{s}\end{array}$ & $\begin{array}{l}\text { Molecular } \\
\text { weight }^{\mathrm{a}}\end{array}$ \\
\hline 21 & 2.34 & 80 & 2.95 & 59,900 \\
\hline 22 & 3.51 & 75 & 4.70 & 58,700 \\
\hline 23 & 4.67 & 70 & 6.60 & 50,700 \\
\hline 24 & 5.83 & 70 & 7.84 & 45,100 \\
\hline
\end{tabular}

\footnotetext{
a Measured on a Knauer membrane osmometer in benzene at $37^{\circ} \mathrm{C}$.
} 
Bulk Copolymerization of 1-Chloro-butadiene with Styrene

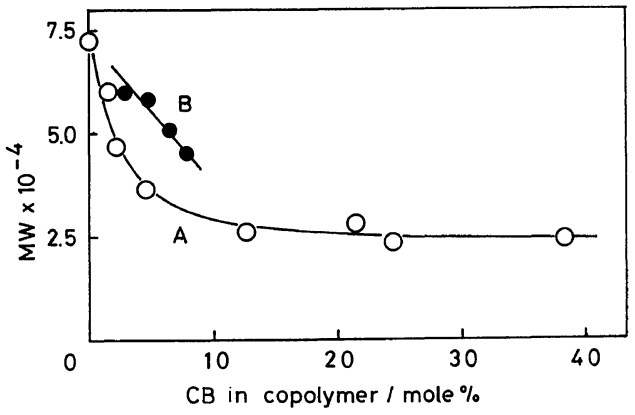

Figure 3. Number-average $\mathrm{MW}$ of poly(CB-co-St)s as a function of $\mathrm{CB}$ content. $\mathrm{A}, \mathrm{MW}$ of the copolymers obtained at low conversions (Table II); B, MW of copolymers obtained at high conversions (Table III).

and we observe the same trends in $\mathrm{MW}$ as in Table II. Since the decrease in CB occurs faster than that of St, the molecular weight of the copolymer should increase as polymerization proceeds. Comparison of the MW in Tables II and III is made in Figure 3 as a function of CB content in poly(CB-co-St). This figure clearly demonstrates the remarkable lowering of the MW of the copolymer with increase in CB content which appears to level off at higher CB content. The effect of conversion on the MW is also displayed in Figure 3. The augmentation of MW with conversion is not ascribable to the polymer reaction between the polymer radical and polymer. If this kind of polymer reaction does occur, crosslinked polymers or at least grafted polymers should be produced. However, no gel was detected in poly(CB-co-St)s and we did not observed any evidence suggesting the formation of grafted polymers in GPC. The number-average molecular weight of poly(CB-co-St) with CB content higher than $40 \mathrm{~mol} \%$ was not available, because the polymer of lower MW than 20,000 (number average) could not be subjected to membrane osmometer measurement effective between $10^{4}$ and $10^{6}$. We may assume that the same trend holds for CB-St copolymers with CB content higher than that of the copolymers listed in Table II.
Table IV. GPC of poly(CB-co-St) $\mathrm{s}^{\mathrm{a}}$

\begin{tabular}{|c|c|c|c|c|}
\hline \multirow{2}{*}{ Sample } & \multirow{2}{*}{$\frac{\begin{array}{c}\text { CB content } \\
\text { in copolymer }\end{array}}{\mathrm{mol} \%}$} & \multicolumn{3}{|c|}{$M W$ from $G^{b} C^{b}$} \\
\hline & & $M_{n}$ & $M_{w}$ & $M_{w} / M_{n}$ \\
\hline 31 & 0 & 24,500 & 43,400 & 1.77 \\
\hline 32 & 2.85 & 17,300 & 30,500 & 1.76 \\
\hline 33 & 5.45 & 15,600 & 26,900 & 1.72 \\
\hline 34 & 8.83 & 12,300 & 20,300 & 1.66 \\
\hline 35 & 18.5 & 11,500 & 17,600 & 1.54 \\
\hline 36 & 28.5 & 9,000 & 13,800 & 1.53 \\
\hline 37 & 43.5 & 8,300 & 12,700 & 1.53 \\
\hline 38 & 100 & 6,400 & 9,800 & 1.53 \\
\hline
\end{tabular}

a Synthesized by bulk polymerization at $80^{\circ} \mathrm{C}$ with AIBN. Conversions were between $15-20 \%$.

b MW refers to that of standard polystyrenes.

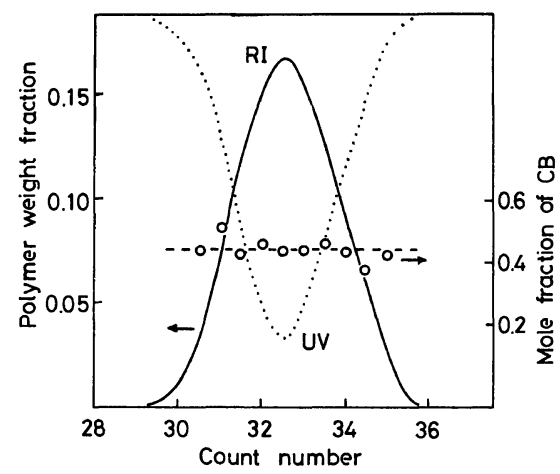

Figure 4. GPC of poly(CB-co-St), sample No. 37 (Table IV); the solid line and the dotted line are RI and UV traces, respectively, and the circles show the pointby-point compositions.

\section{Molecular Weight Distribution by GPC}

The copolymers were subjected to GPC measurements to obtain MWD data. Table IV shows the results of GPC measurements on poly(CB-co-St)s obtained by polymerization at $80^{\circ} \mathrm{C}$. In the last column, the ratios of $M_{w}$ and $M_{n}$ are tabulated. With an increase in CB content in the copolymer, the ratio decreased from 1.77 for poly(St) to 1.53 for poly $(\mathrm{CB})$. When $\mathrm{CB}$ content was higher than $\mathrm{ca} .10 \%$, the ratio appeared to reach an asymptotic value which remained constant up to the CB homopolymer.

In Figure 4, GPC curves and the point-bypoint compositions of poly $(\mathrm{CB}-\mathrm{co}-\mathrm{St})$ are 
shown. Both RI and UV traces were unimodal; thus the ratio $M_{w} / M_{n}$ can be an appropriate index representating the MWD of poly(CB-co-St). The point-by-point composition, i.e., the average composition of the copolymer at each count, was calculated from RI and UV traces of GPC. This composition remained constant regardless of MW (count number). Though the copolymerization rate and $\mathrm{MW}$ of the copolymer depended heavily on CB concentration in the monomer feed, the invariance of that composition suggests that random copolymerization occurs between $\mathrm{CB}$ and St. $^{15,16}$

\section{Behavior of $C B$ in Radical Copolymerization}

The $Q$ value and MRR of CB show it to be a conjugated monomer and more reactive that St. We also observed lowerings of rate (Figure 1), MW (Table II and Figure 3), and the ratio of $M_{w}$ and $M_{n}$ from GPC (Table IV) with increase in CB content. These lowerings were remarkably pronounced at very low CB concentrations. The rate decrease can be interpreted by the active crossover termination.

To elucidate the factors influencing the MW of poly(CB-co-St), a few considerations were made. As a first approximation, we assume that $\mathrm{CB}$ is acting as a chain-transfer agent during polymerization to determine molecular weight. In that case, the following Mayo equation should be applicable ${ }^{17}$ :

$$
\frac{1}{D P}=\frac{1}{D P_{0}}+C_{\mathrm{s}} \cdot \frac{[\mathrm{CB}]}{[\mathrm{St}]}
$$

where $D P$ and $D P_{0}$ are the degrees of polymerization of polymers produced in the presence and absence of $\mathrm{CB}$, respectively, and $C_{\mathrm{s}}$ is a chain-transfer constant. This very simple situation was not the case for the data in Table II, since the Mayo plot (1/DP vs. [CB]/[St]) did not show any linearity.

The second approach assumes a case such that both transfer to the monomer and termination are important, i.e., the following reac- tions are considered to determine the molecular weight:

chain transfer to monomer:

$$
\begin{aligned}
& -\mathrm{M}_{1} \cdot+\mathrm{M}_{1} \rightarrow \mathrm{P}+\mathrm{M}_{1} \cdot k_{t_{\mathrm{m} 11}} \\
& -\mathrm{M}_{1} \cdot+\mathrm{M}_{2} \rightarrow \mathrm{P}+\mathrm{M}_{2} \cdot k_{t_{\mathrm{m} 12}} \\
& -\mathrm{M}_{2} \cdot+\mathrm{M}_{1} \rightarrow \mathrm{P}+\mathrm{M}_{1} \cdot k_{t_{\mathrm{m} 21}} \\
& -\mathrm{M}_{2} \cdot+\mathrm{M}_{2} \rightarrow \mathrm{P}+\mathrm{M}_{2} \cdot k_{t_{\mathrm{m} 22}}
\end{aligned}
$$

termination:

$$
\begin{array}{ll}
-\mathrm{M}_{1} \cdot+-\mathrm{M}_{1} \cdot \rightarrow \mathrm{P} & k_{t_{1}} \\
-\mathrm{M}_{1} \cdot+-\mathrm{M}_{2} \cdot \rightarrow \mathrm{P} & k_{t_{12}} \\
-\mathrm{M}_{2} \cdot+-\mathrm{M}_{2} \cdot \rightarrow \mathrm{P} & k_{t_{2}}
\end{array}
$$

where $M_{1}$ refers to St, $M_{2}$ to $C B$ and $P$ to a dead copolymer. This assumption may be reasonable, since all poly(CB-co-St)s were produced in bulk where no transfer reagents were present except monomers. The number-average degree of polymerization is expressed as

$$
P_{n}=-\frac{\mathrm{d}[\mathrm{M}]}{\mathrm{d} t} / \frac{\mathrm{d}[\mathrm{P}]}{\mathrm{d} t}=-\frac{\mathrm{d}\left(\left[\mathrm{M}_{1}\right]+\left[\mathrm{M}_{2}\right]\right)}{\mathrm{d} t} / \frac{\mathrm{d}[\mathrm{P}]}{\mathrm{d} t}
$$

and

$$
\frac{\mathrm{d}[\mathrm{P}]}{\mathrm{d} t}=R_{t_{\mathrm{m}}}+\frac{1}{2} R_{t}
$$

where $R_{t_{\mathrm{m}}}$ is the rate of monomer transfer (eq 3-6) and $R_{t}$, that of termination (eq 7-9).

In radical polymerizations, monomer-transfer reactions are not considered important except for some monomers, such as allylic monomer ${ }^{18}$ and $\alpha$-methylstyrene. ${ }^{19}$ However, a remarkable occurrence of crossover monomer-transfer reactions ${ }^{12}$ (eq 4 and 5) seems a possible explanation for the drastic decrease in MW by CB. GPC data in Table IV, however, show this not to be the case. When the combination reaction is predominant in termination and transfer reactions are insignificant, $M_{w} / M_{n}$ is $1.5,{ }^{20,21}$ and if transfers to monomer are dominating, $M_{w} / M_{n}=2.0 .^{21,22}$ Because $M_{w} / M_{n}$ approached 1.5 with increase in CB 
concentration and combination was preferred to disproportionation in the copolymerization, ${ }^{23}$ monomer-transfer reactions seem improbable. Consequently, $R_{t_{\mathrm{m}}}$ can be neglected in eq 11 .

With the substitution of $M_{1}$ and $M_{2}$ by $S t$ and $\mathrm{CB}$, respectively, eq 11 becomes

$$
\begin{aligned}
2 \frac{\mathrm{d}[\mathrm{P}]}{\mathrm{d} t} & =R_{t} \\
& =k_{t_{1}}[\mathrm{St} \cdot]^{2}+k_{t_{12}}[\mathrm{St} \cdot][\mathrm{CB} \cdot]+k_{t_{2}}[\mathrm{CB} \cdot]^{2}
\end{aligned}
$$

where $\mathrm{St} \cdot$ and $\mathrm{CB} \cdot$ designate the propagating radicals ending in $\mathrm{St}$ and $\mathrm{CB}$ units, respectively.

Combining eq 1, 10 and 12, we obtain

$$
\frac{1}{\bar{P}_{n}}=\frac{R_{i}^{1 / 2}\left(r_{1}^{2} \delta_{1}^{2}\left[\mathrm{M}_{1}\right]^{2}+2 \phi r_{1} r_{2} \delta_{1} \delta_{2}\left[\mathrm{M}_{1}\right]\left[\mathrm{M}_{2}\right]+r_{2}^{2} \delta_{2}^{2}\left[\mathrm{M}_{2}\right]^{2}\right)^{1 / 2}}{2\left(r_{1}\left[\mathrm{M}_{1}\right]^{2}+2\left[\mathrm{M}_{1}\right]\left[\mathrm{M}_{2}\right]+r_{2}\left[\mathrm{M}_{2}\right]^{2}\right)}
$$

where the stationary state is reasonably assumed, i.e.,

$$
R_{i}=R_{t}
$$

Equation 13 is transformed to eq 14 using $X=$ $\left[\mathrm{M}_{2}\right] /\left[\mathrm{M}_{1}\right]=[\mathrm{CB}] /[\mathrm{St}]$.

$\frac{1}{\bar{P}_{n}}=\frac{R_{i}^{1 / 2}\left(\delta_{1}^{2}+2 \phi \frac{r_{2}}{r_{1}} \delta_{1} \delta_{2} X+\left(\frac{r_{2}}{r_{1}}\right)^{2} \delta_{2}^{2} X^{2}\right)^{1 / 2}}{2[\mathrm{St}]\left(1+\frac{2}{r_{1}} X+\frac{r_{2}}{r_{1}} X^{2}\right)}$

The molecular weight decrease was pronounced at very low [CB], below ca. $5 \mathrm{~mol} \%$ as seen from Figure 3 and Table II. Consequently, the second-order term of $X$ may be neglected as a first-order approximation. Thus, eq 14 is reduced to eq 15 :

$$
\frac{1}{\bar{P}_{n}}=\frac{R_{i}^{1 / 2}\left(\delta_{1}^{2}+2 \frac{r_{2}}{r_{1}} \frac{k_{t_{12}}}{k_{11} k_{12}} X\right)^{1 / 2}}{2[\mathrm{St}]\left(1+\frac{2}{r_{1}} X\right)}
$$

This equation is amenable to calculation, when $R_{i}$ is assumed not to change between St polymerization and copolymerizations of $\mathrm{St}$ with CB. By that assumption, we obtain $R_{i}^{1 / 2} / 2[\mathrm{St}]=$ $3.92 \times 10^{-5}$. The value of $r_{1}$ and $r_{2}$ are available, and $k_{11}\left(=k_{p}\right.$ of $\left.\mathrm{St}\right)$ and $\delta_{1}$ are found in the literature. ${ }^{24}$ Then, eq 15 becomes eq 16 :

$$
\begin{aligned}
& \frac{1}{\bar{P}_{n}}=\frac{3.92 \times 10^{-5}\left(1350+4.77 \times 10^{-2} \alpha X\right)^{1 / 2}}{1+7.30 X} \\
& \text { where }
\end{aligned}
$$

$$
\alpha=\frac{k_{t_{12}}}{k_{22}}
$$

The plot according to eq $16\left(\bar{P}_{n} v s . X\right)$ is shown in Figure 5. By computer simulation, we found the optimum value of $\alpha$ to be $8.0 \times$ $10^{6}$ in the range of $X$ from 0 to 0.50 . The solid line $A$ is that calculated using $\alpha=8.0 \times 10^{6}$. At a higher $X$ region, this $\alpha$ value does not satisfy the experimental results. There, $X^{2}$ might not be negligible, and in that case, not eq 15 but eq 14 should be used and is converted to the following equation:

$$
\frac{1}{\bar{P}_{n}}=\frac{3.92 \times 10^{-5}\left(1350+4.77 \times 10^{-2} \alpha X+2.82 \beta X^{2}\right)^{1 / 2}}{1+7.30 X+6.13 X^{2}}
$$

where $\alpha=k_{t_{12}} / k_{22}$ and $\beta=\delta_{2}^{2}=K_{t_{2}} / k_{22}^{2}$.

By the computer simulation technique, the optimum set of $\alpha$ and $\beta$ was found:

$$
\begin{aligned}
& \alpha=\frac{k_{t_{12}}}{k_{22}}=7.0 \times 10^{6} \\
& \beta=\delta_{2}^{2}=\frac{k_{t_{2}}}{k_{22}^{2}}=50 \mathrm{~mol} \mathrm{~s}^{-1}
\end{aligned}
$$




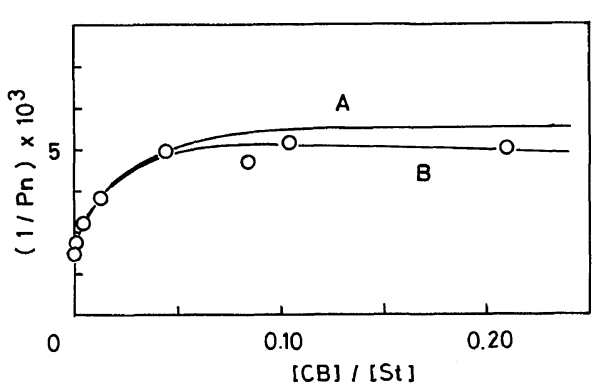

Figure 5. Relationship according to eq 16 or 17 ; circles are experimental points; solid line A, calculated from $\alpha=$ $8.0 \times 10^{6}$ (eq 16); solid line $\mathrm{B}$, calculated from $\alpha=$ $7.0 \times 10^{6}$ and $\beta=50 \mathrm{~mol} \mathrm{~s}^{-1}$.

The solid line B in Figure 5 is that calculated using these values.

As far as we know, only one report ${ }^{25}$ treats the determination of rate constants, $k_{p}$ and $k_{t}$, of 1 -substituted butadiene derivatives. According to that report, these constants were not so much different from those of St as supposed by the large difference in $Q$ values, i.e., $Q=1.0$ (St) and $Q=3.19-9.57$ (four 1-substituted butadienes). In the case of 1-acetoxybutadiene $(Q=3.19) k_{t}$ is even larger than that of $\mathrm{St}^{25}$ No numerical value of $k_{22}$ is now available, but from the value, $\alpha=7$ or $8 \times 10^{6}, k_{t_{12}}$ can be very large and, as such determines the MW of poly(CB-co-St) when $X=[\mathrm{CB}] /[\mathrm{St}]$ is low. At a higher $\mathrm{CB}$ concentration, termination between the chain ending in $\mathrm{CB}$ unit becomes more important. From these considerations, we estimate that the drastic decrease in rate and MW are due to the large crossover termination rate constant $\left(k_{t_{12}}\right)$ between St and CB polymer radicals.

Crossover termination rate constant $\left(k_{t_{12}}\right)$ has so far been discussed through:

$$
\phi^{2}=k_{t_{12}}^{2} / k_{t_{1}} k_{t_{2}}
$$

This parameter is determined from the copolymerization rate (eq 1). Some established results $^{23}$ show that $\phi$ is equal to unity for the monomer pair where the product $r_{1} \cdot r_{2}$ is nearly unity. The product $r_{1} \cdot r_{2}$ is regarded as a parameter related to the alternating tendency of a given monomer pair, i.e., the smaller $r_{1} \cdot r_{2}$, the more alternating is the propagation. In accord with this tendency, crossover termination may be more predominant $(\phi>1.0)$ in the monomer pair when $r_{1} \cdot r_{2}$ is smaller than unity. ${ }^{12,23}$ In the copolymerization of St and $\mathrm{CB}$, the two kinetic parameters $\alpha$ and $\beta$ were evaluated. The parameter $\phi$ can be expressed by $\alpha$ and $\beta$ as follows:

$$
\phi^{2}=\frac{k_{t_{12}}^{2}}{k_{t_{1}} k_{t_{2}}}=\frac{\alpha^{2}}{k_{t_{1}} \beta}
$$

Using $k_{t_{1}}=8.91 \times 10^{7} 1 \mathrm{~mol}^{-1} \mathrm{~s}^{-1},{ }^{24} \alpha=7.0 \times$ $10^{6}$, and $\beta=50 \mathrm{mols}^{-1}, \phi$ is calculated

$$
\phi \simeq 100 \gg 1.0
$$

This value should be considered only as semiquantitative, since both $\alpha$ and $\beta$ were obtained by the curve-fitting method on MW data. However, the large $\phi$ again supports the assumption that $k_{t_{12}}$ is very large for the St-CB pair.

It is noticeable that since the molecular weight of poly(CB-co-St) seems to be determined by the chemical nature of the two monomers, it depends entirely on the $[\mathrm{CB}] /[\mathrm{St}]$ ratio and cannot be independent of the copolymer composition. Therefore, when we know the composition of CB-St copolymer, its molecular weight is already determined by eq 17 , and because molecular weight decreases with an increase in CB content, it would be very difficult to synthesize high-CB-content poly $(\mathrm{CB}-\mathrm{co}-\mathrm{St})$ of high molecular weight by bulk polymerization.

Acknowledgment. This work was supported in part by a Grant-in-Aid for Scientific Research from the Ministry of Education, Science, and Culture (Grant No. 57470078).

\section{REFERENCES}

1. S. Yamashita, S. Atomori, S. Kohjiya, and T. Miyagawa, J. Appl. Polym. Sci., 17, 3049 (1973).

2. S. Yamashita, M. Tamura, J. Terada, and S. Kohjiya, Rubber Chem. Technol., 50, 364 (1977). 
3. S. Yamashita, K. Sando, and S. Kohjiya, J. Appl. Polym. Sci., 23, 1951, 1963 (1979).

4. S. Kohjiya, Y. Hongu, M. Tamura, and $\mathrm{S}$. Yamashita, Polym. Bull., 6, 209 (1981).

5. S. Kohjiya, H. Takeuchi, K. Kawamoto, and S. Yamashita, Bull. Chem. Soc. Jpn., 54, 3245 (1981).

6. V. L. Heasley and B. R. Lais, J. Org. Chem., 33, 2571 (1968):

7. A. Winston and P. Wichacheewa, Macromolecules, $\mathbf{6}$, 200 (1973).

8. S. Kohjiya, K. Iwata, S. Yamashita, T. Miyamoto, and H. Inagaki, Polym. J., 15, 869 (1983).

9. C. Walling, J. Am. Chem. Soc., 71, 1930 (1949).

10. E. Arlman, H. Melville, Proc. R. Soc. London, Ser. A., 200, 337 (1950).

11. S. Kohjiya, S. Fujiwara, K. Fujii, and S. Yamashita, Makromol. Chem., 183, 163 (1982).

12. C. H. Bamford, W. G. Barb, A. D. Jenkins, and P. F. Onyon, "The Kinetics of Vinyl Polymerization by Radical Mechanisms," Butterworths, London, 1958, Chapter 5.

13. X. C. Bagdasaryan, "Theory of Radical Polymerization," U.S.S.R. Science Academy Publisher, Moscow, 1959, Chapter 4.

14. T. Alfrey, Jr., and L. J. Young, "Copolymerization," ed. by G. E. Ham, Interscience, New York, N. Y., 1961, Chapter 2 and Appendix B.

15. S. Teramachi and Y. Kato, Macromolecules, 4, 54 (1971).

16. H. Inagaki, Kaigai Kobunshi Kenkyu, 25, 211 (1979).

17. Reference 12, Chapter 6.

18. P. D. Bartlett and F. A. Tate, J. Am. Chem. Soc., 75, 91 (1953).

19. A. Rudin, M. C. Samanta, and B. M. E. van der Hoff, J. Polym. Sci., Polym. Chem. Ed., 17, 493 (1979).

20. G. Gee and H. W. Melville, Trans. Faraday Soc., 40, 240 (1944).

21. A. Nakajima and M. Hosono, "Molecular Properties of Polymers," Kagaku Dojin, Kyoto, 1969, Chapter 3.

22. T. Higashimura, Yu. Imanishi, T. Fukushima, Yo. Imanishi, and S. Okamura, Kobunshi Kagaku, 22, 205 (1965).

23. J. C. Bevington, "Radical Polymerization," Academic Press, London, 1961, Chapter 6.

24. M. S. Matheson, E. E. Auer, E. B. Bevilacqua, and E. J. Hart, J. Am. Chem. Soc., 73, 1700 (1951).

25. M. Kamachi, H. Umethani, and S. Nozakura, Polym. Prepr., Jpn., 32, 1103 (1983). 